Methods to quantify in vivo phagocytic uptake and opsonization of live or killed microbes in Drosophila melanogaster

Samuel Liégeois ${ }^{1,2 *}$, Wenhui Wang ${ }^{1}$, and Dominique Ferrandon ${ }^{1,2}$

1) Sino-French Hoffmann Institute, Guangzhou Medical University, Guangzhou, P. R. China

2) Université de Strasbourg, CNRS, M3I UPR 9022, F-67000 Strasbourg, France

* To whom correspondence should be addressed (s.liegeois@unistra.fr)

Running Head:

Drosophila in vivo and ex vivo phagocytosis assays 


\section{i. Summary/Abstract}

Here we describe different phagocytosis assays in Drosophila, using various killed or live microbes (bacteria and fungi). Different ex vivo and in vivo approaches are shown, to quantify larval and adult phagocytosis of microorganisms by hemocytes. We also explain how to perform an in vivo opsonization assay. Altogether, these protocols represent a useful range of tools to the researcher interested in the detailed analysis of phagocytosis in the context of the study of host-pathogen relationships.

\section{ii. Key Words}

Opsonization, phagocytosis, Drosophila, larva, hemocytes, bacteria, fungi, yeast, infection, pHrodo

\section{Introduction}

The insect immune system comprises several arms, including a systemic humoral response, the melanization response triggered by proteolytic cascades, and the cellular immune response (1). While much emphasis has been placed on the study of the humoral immune response in the past 30 years because of its efficacy against most microbes, the cellular immune response is much less understood in molecular terms. In Drosophila, blood cells are called hemocytes, and most of them are phagocytotic macrophage-like cells named plasmatocytes.

Two waves of hematopoiesis occur during Drosophila development. The embryonic/larval lineage originates from the head mesoderm of the embryo, differentiates in the embryo, and subsequently expands in the larva. The progenitor-based lymph gland lineage originates in the dorsal mesoderm of the embryo and differentiates in the late larva (2). Hemocytes of both lineages persist through pupal development into the adult. During the larval instars, hemocytes derived from embryogenesis spread throughout the animal and are found in two 
locations: a subset of them circulates in the hemolymph and the rest are attached to the body wall in sessile pools. At the end of the third instar larval stage, called the wandering stage, ecdysone signaling induces dispersal and activation of sessile hemocytes upon pupariation, and this facilitates tissue remodeling during metamorphosis. Several hundred blood cells are made in the embryo. This number expands through the larval stages to more than 5000 hemocytes during the pupal stage. At this point, there is a high demand for blood cells to accommodate the extensive histolysis and tissue remodeling occurring during metamorphosis. The total number of hemocytes in the adult ranges between 1000 and 2000 cells per larva (3). Functionally, phagocytosis does not seem to play a preponderant role in the host defense against Gram-negative bacteria in systemic infection models. It, however, plays an essential role in controlling bacteria that evade the digestive tract (4-6). As regards Gram-positive bacteria and fungi, phagocytosis fulfills a more important function in host defense as its ablation leads to an enhanced sensitivity to these systemic infections $(7,8)$. It may also represent a remaining protection when the humoral immune response is deficient. Investigators initially relied on semi-quantitative assays or ex vivo quantitative assays (9). A common feature of these studies is that they used fluorescently-labeled killed microbes, usually Staphylococcus aureus as a Gram-positive bacterium, Escherichia coli as a Gramnegative bacterium, and zymozan particles to mimic fungal cells. The fluorescence of noninternalized bacteria was then quenched by adding Trypan blue, thereby allowing the visualization of engulfed bacteria only $(4,10,11)$ (See Note 10). A positive control consisted in saturating the phagocytic apparatus by the prior injection of "latex" beads (actually often polystyrene beads), a strategy that can also be used for other insects $(10,12,13)$. A second possible control would have been to use hemocyte-depleted flies $(14,15)$. A major difficulty for establishing quantitative assays in adults is the low number of plasmatocytes that can be retrieved, as most of them are sessile. In contrast, it is easy to obtain hundreds of hemocytes 
by just bleeding a single larva. As it is difficult to inject Drosophila larvae, ex vivo assays have been favored. One advantage of using killed microbes is that one can measure the host response in the absence of active interference from the pathogen. However, microbes have developed several strategies to elude phagocytosis, either by blocking it directly or by avoiding detection. Thus, when studying host-pathogen relationships, it is critical to also monitor the uptake of live microorganisms.

In this Chapter, we provide descriptions of several techniques currently used in the field to quantify phagocytosis that we routinely use in the laboratory $(8,16)$. We selected as examples infection models currently under investigation in the team (the Gram-negative bacteria Serratia marcescens and Pseudomonas aeruginosa, the mold Metarhizium anisopliae and the yeast Candida glabrata) but the protocols described here can be generalized to other microbes. We shall describe on the one hand methods to quantify microorganism internalization using either ex vivo or in vivo assays and on the other hand a method to study the coating of a particle with proteins that facilitate phagocytosis of the particle by macrophages, a process called opsonization. These different techniques partially overlap and we have therefore decided to detail the common steps in the form of modules (Figure 1), detailed in Part 3 (Methods). First, development of antibodies (3.1) and preparation of microorganisms (3.2) are described. Then, collection of hemocytes and preparation of tissues (3.3) can be done at the beginning of ex vivo phagocytosis assays (3.4), or after incubation with microbes for in vivo assays (3.5) and opsonization assays (3.6). Of note, the in vivo technique necessitates the injection of microorganisms, a procedure that is straightforward in adults yet challenging in Drosophila larvae (reported in section 3.5). The opsonization assay involves a more complex procedure (section 3.6, Figure 1B). At the end of the different assays, when using nonlabelled microbes, the in/out differential immunostaining procedure can be performed to reveal whether the microorganisms have been internalized (3.7). It is not the case for one of 
the ex vivo phagocytosis assays that relies on pHrodo-labeled microorganisms. Finally, the last step described is the sample analysis by microscopy (3.8).

These methods allow studying phagocytosis not only from the standpoint of the host, but within the more meaningful context of host-pathogen relationships in which pathogens attempt to elude or neutralize the host immune response $(6,16-18)$. 


\section{Materials}

\subsection{Drosophila and microbial strains; culture conditions:}

1. Two to seven days old Drosophila melanogaster females from the following genotypes:

wild-type $w^{A 5001}$, homozygous recombinant HMLdelta-GAL4,UAS-GFP flies constitutively expressing GFP in hemocytes (19) and Tep4 mutants (Bloomington stock \#15936), checked for the absence of known contaminants $(20,21)$, and conventionally reared at $25^{\circ} \mathrm{C}$

2. Serratia marcescens wild-type Db11; overnight culture in LB medium at $37^{\circ} \mathrm{C}$

3. Pseudomonas aeruginosa wild-type PA14; overnight culture in $\mathrm{BHB}$ medium at $37^{\circ} \mathrm{C}$

4. Metarhizium anisopliae wild-type ARSEF 2575; cultured on PDA (Potato Dextrose Agar) plates at $25^{\circ} \mathrm{C}$ for 7 to 14 days

5. Candida glabrata wild-type ATCC 2001; overnight culture in YPD medium at $30^{\circ} \mathrm{C}$

6. Any bacterial or fungal strain of interest

\subsection{Material for microbial solutions}

1. GeneQuant pro Spectrophotometer (Amersham Biosciences)

2. Eresco 42 MF2, Cegelec (Source: Beryllium) generator of X-rays (for exposure to X-rays)

3. Precision wipes (Kimtech Science)

\subsection{Material for injection:}

1. Short capillaries: 3.5" (Drummond)

2. Long capillaries: 7" (Drummond)

3. Adaptor for long capillaries with a syringe after a flexible tube (in order to fill the capillary with a large volume, about $20 \mu \mathrm{L})($ see Figure 2F)

4. Flaming/Brown micropipette puller (Sutter Instrument, Model P-97)

5. Nanoject II Auto-Nanoliter Injector (Drummond) 


\subsection{Sample preparation for microscopy}

1. Dumont \#5 tweezers

2. Diagnostic Microscope Slides Epoxy 8-Wells 6 mm Black (Thermo Scientific)

3. Plastic box with a layer of wet paper to use as a humid chamber

4. Fluorescent Zeiss Axioscope 2 microscope (Carl Zeiss, Göttingen, Germany)

\subsection{Antibodies:}

Primary antibodies detecting bacterial or fungal strains used for infection:

1. Rabbit antibodies against UV-killed wild-type Pseudomonas aeruginosa PA14 by Covalab; Dilution 1/200 (16) (see Note 1)

2. Antibodies raised against whole UV-killed Candida (from Karl Kuchler, Vienna) used against C. glabrata (8); Dilution 1/1000 (see Note 1)

3. Rabbit antibodies against UV-killed wild-type M. anisopliae ARSEF 2575 (From Wenhui Wang, SFHI, China); Dilution 1/1000 (see section 3.1)

4. Mouse monoclonal antibodies against the phagocytosis receptor Nimrod, expressed in hemocytes (P1, from István ANDÓ, Hungary) (22)

Matching secondary fluorescently labeled antibodies for detection:

5. FITC-labeled goat anti-rabbit secondary antibodies

6. Cy3-labeled goat anti-rabbit secondary antibodies

\subsection{Media and buffers}

1. Lysogeny Broth LB-Miller: Tryptone $10 \mathrm{~g} / \mathrm{L}$, Yeast Extract $5 \mathrm{~g} / \mathrm{L}, \mathrm{NaCl} 10 \mathrm{~g} / \mathrm{L}$ in $\mathrm{H} 20$

2. Bushnell Haas Broth (BHB): MgSO4 0.2g/L, CaCl2 0.02 g/L, KH2PO4 1g/L, K2HPO4 $1 \mathrm{~g} / \mathrm{L}, \mathrm{NH} 4 \mathrm{NO} 31 \mathrm{~g} / \mathrm{L}, \mathrm{FeCl} 30.05 \mathrm{~g} / \mathrm{L}$ in $\mathrm{H} 20$ 
3. Potato Dextrose Agar (PDA): Dextrose 20g/L, Potato starch 4g/L, Agar 15g/L in H20

4. Yeast Extract Peptone Dextrose (YPD): Peptone 20g/L, Dextrose 20g/L, Yeast Extract $10 \mathrm{~g} / \mathrm{L}$ in $\mathrm{H} 20$

5. Phosphate-buffered saline (PBS): $\mathrm{NaCl} \mathrm{8g/L,} \mathrm{KCl} \mathrm{0.2g/L,} \mathrm{Na2HPO4} \mathrm{1.44g/L,} \mathrm{KH2PO4}$ $0.24 \mathrm{~g} / \mathrm{L}$ in $\mathrm{H} 20(\mathrm{pH}=7.4)$

\subsection{Reagents}

1. Freund's Adjuvant: $0.85 \mathrm{~mL}$ paraffin oil and $0.15 \mathrm{~mL}$ mannide monooleate (Incomplete)

+/- $1 \mathrm{mg}$ of Mycobacterium tuberculosis (H37Ra, ATCC 25177), heat killed and dried (Complete)

\section{2. $0.1 \mathrm{M} \mathrm{Na} 2 \mathrm{CO} 3$}

3. 16\% Paraformaldehyde aqueous solution

4. Bovine Serum Albumin

5. Tween 20 (Polysorbate 20)

6. Triton $\mathrm{X}-100$

7. Vectashield Antifade Mounting Medium with DAPI (Vector Laboratories)

8. Trypan blue $0.4 \%$ solution in PBS

9. CML Latex Beads, $4 \% \mathrm{w} / \mathrm{v}, 0.3 \mu \mathrm{m}$ (ThermoFisher Scientific)

10. Fluorescein-5-isothiocyanate (FITC)

11. pHrodo $^{\mathrm{TM}}$ Red succinimidyl ester (Invitrogen)

12. pHrodo $^{\mathrm{TM}}$ Red E. coli BioParticles ${ }^{\mathrm{TM}}$ Conjugate for Phagocytosis (Invitrogen)

\section{Methods}




\subsection{Development of antibodies against Metarhizium anisopliae}

1. M. anisopliae ARSEF 2575 is plated on PDA plate at $25^{\circ} \mathrm{C}$ for one week.

2. $15 \mathrm{~mL}$ PBS with $0.01 \%$ Tween 20 are added to the plate, mixed, and a spore suspension is prepared by filtration through a precision wipe.

3. The suspension is transferred in a Petri dish on ice under a UV-C intensity of $1200 \mu \mathrm{W} / \mathrm{cm}^{2}$ for 1 hour (using a 15W UV lamp at a distance of $10 \mathrm{~cm}$ ), allowing to kill microbes (see Note 2).

4. A small volume of UV-killed spore solution is plated to confirm the spores are dead.

5. Immunization was done by multiple subcutaneous injections ( 4 times), each time with 250 million UV-killed spores, emulsified with complete Freund adjuvant at day 1, and emulsified with incomplete Freund adjuvant at day 14, 28 and 42, in the back of four New Zealand rabbits.

6. At day 49, animals were sacrificed, and their total blood was collected from heart. $1 \mathrm{~mL}$ of non-immunized rabbit serum was collected as a negative control.

7. Affinity purification of antisera has been done using Protein G chromatography, and $50 \mathrm{mg}$ of antibodies were purified from each immunized rabbit.

\subsection{Microbe solution preparation (killed and fluorescently-labeled microbes, or live} ones)

Killing can be achieved on fungal or bacterial cultures grown during the exponential phase (see Note 2):

- by chemical fixation in PBS with $4 \%$ paraformaldhehyde for $16 \mathrm{~h}$ at room temperature - by heating the cultures at $65^{\circ} \mathrm{C}$ for $60 \mathrm{~min}$

- by administration of a UV-C light intensity of $1200 \mu \mathrm{W} / \mathrm{cm}^{2}$ for 1 hour (see section 3.1.3) 
- by exposing cultures to X-rays at $2 \mathrm{krad} / \mathrm{min}(20 \mathrm{~Gy} / \mathrm{min})$ for $6 \mathrm{~h}$ corresponding to a total exposure dose of $720 \mathrm{krad}$.

Killed cells are washed twice in PBS and plated to ensure nonviability, on LB, BHB, PDA or YPD agar plates, according to the microbial species (see section 2.1).

2. Staining of killed microbes can be done using FITC or pHrodo. The principle of pHrodo is that this dye is nonfluorescent at neutral $\mathrm{pH}$ and exhibits increasing fluorescence (red or green) as the $\mathrm{pH}$ becomes more acidic, such as that encountered in the mature phagolysosome. Thus, there is no need for quenching and only internalized bacteria that are being located in the final degradation compartment are visualized (see Note 3). FITC labeling is done by washing microbes $\left(10^{8}\right.$ cells $\left./ \mathrm{mL}\right)$ with a $0.1 \mathrm{M}$ sodium carbonate $(\mathrm{Na} 2 \mathrm{CO} 3)$ solution at $\mathrm{pH}=9.2(\mathrm{pH}$ is important) with $0.05 \%$ Tween 20 followed by incubation in the same solution with $100 \mu \mathrm{g} / \mathrm{mL}$ FITC, overnight at $4^{\circ} \mathrm{C}$ or $2 \mathrm{~h}$ at room temperature, in the dark (from a stock solution of $2 \mathrm{mg} / \mathrm{mL}$ FITC in DMSO). Wash extensively with PBS $+0.01 \%$ Tween 20 (four times with an equal volume) Check the final cell suspension microbe concentration by direct counting using a hemocytometer. Microbes can be labeled with pHrodo, following the instructions from Invitrogen. Solutions at $5.10^{10}$ bacteria $/ \mathrm{mL}$ in PBS (dose used for Pseudomonas aeruginosa PA14) are aliquoted and kept frozen at $-20^{\circ} \mathrm{C}$ before use (see Note 4).

3. Live microbes: Fresh overnight cultures are used for live assays. Optical density (OD) at $600 \mathrm{~nm}$ was adjusted to a value of 20 in PBS for the bacteria P. aeruginosa or S. marcescens; for fungi, 4000 Candida glabrata yeasts in a $0.01 \%$ Tween 20 solution in PBS (see Note 5) are injected whereas 3500 M. anisopliae spores are injected $\left(69 \mathrm{~nL}\right.$ of a $5.10^{7}$ spores $/ \mathrm{mL}$ solution). 


\subsection{Animal bleeding and dissection for visualization of internalized bacteria on tissue-resident plasmatocytes (see Note 6)}

1. Larvae are bled by dissecting it with tweezers and shaking it on the solution into the wells, before discarding the cadaver: hundreds of hemocytes will be found in each well (see Note 7). 2. Adult hemolymph collection needs more preparation. First, cut a small part of the abdomen, using tweezers (a hole is enough). Second, take the adaptor described in section 2.3.3 and fill a long capillary with $20 \mu \mathrm{L}$ PBS, that can be injected in the thorax: this PBS will go out from the opened abdomen with hemocytes. Take this solution into a well from an 8-well microscope slide that was previously filled with $20 \mu \mathrm{L}$ of PBS (see Figure 2F).

3. Adult tissue dissection consists in removing the head and the thorax, keeping the fly abdomen, dissecting the abdomen from the ventral side, and then removing all internal tissues (reproductive and digestive tract). Most hemocytes will be still present after dissection, lining the respiratory epithelia of the head and thorax (23).

\subsection{Ex vivo phagocytosis assays (see Note 8)}

1. Place a volume of $20 \mu \mathrm{L}$ of a microbial solution (either killed or live microbes) in each well of an 8-well microscope slide.

2. Bleed one animal (a third instar wandering larva or an adult fly) per well (see Note 9)

3. Incubate for 40 minutes in a humid chamber (see Note 10).

4. For FITC-labeled particles, mount the slides on a 0.4\% trypan blue solution in PBS (see Note 11), and incubate for 10-30 minutes before observation by microscopy (See 3.8.3).

4. For pHrodo-labeled particles, wash for 10 minutes in $20 \mu \mathrm{L}$ of a $0.5 \mu \mathrm{g} / \mathrm{mL}$ DAPI solution in PBS, mount the slides on PBS, and analyze by live microscopy (See 3.8.3). For non-labeled microbes, follow the steps described for sample preparation for microscopy (see 3.6) 


\subsection{In vivo phagocytosis assays}

1. Inject third instar wandering larvae (see Note 12) or adult flies with $27.6 \mathrm{~nL}$ of a microbial solution (either killed or live microbes), using a Nanoject apparatus (Drummond).

2. Incubate for $60 \mathrm{~min}$ (see Note 13).

3. Bleed one larva (see Note 9 and 14) or dissect tissue from one fly per well, into $20 \mu \mathrm{L}$ of PBS on an 8-well microscope slide.

4. Incubate for $25 \mathrm{~min}$ in a humid chamber (see note 10).

5. For pHrodo-labeled particles, wash for 10 minutes in $20 \mu \mathrm{L}$ of a $0.5 \mu \mathrm{g} / \mathrm{mL}$ DAPI solution in PBS, mount the slides on PBS, and analyze by live microscopy (See 3.8.3). For non-labeled microbes, follow the steps described on section $\mathbf{3 . 8}$.

\subsection{Opsonization assay on live microbes (Figure 1B)}

The opsonization assay involves a complex procedure. First, the live microbes are incubated in vitro with cell-free hemolymph collected from larvae of either wild-type or mutant for the gene encoding a potential opsonin. The microorganisms will become coated with the opsonins if present in the collected hemolymph. These potentially opsonized bacteria are then injected in opsonin-deficient larvae and the phagocytic index is measured on bled plasmatocytes after an incubation period. If the mutant gene product is really required for opsonization then the phagocytic index will be lower when the larvae have been injected with the microorganisms incubated first with the opsonin-deficient hemolymph than when injecting the opsonized microbes incubated at the first step of the procedure with wild-type hemolymph (16).

1. Prepare $150 \mu \mathrm{L}$ of a microbial solution in a $1.5 \mathrm{~mL}$ microtube (see Note 3).

2. Bleed 20 third instar larvae directly into the bacterial solution (see Note 15).

3. Incubate at room temperature for 30 to $45 \mathrm{~min}$ (to allow potential opsonization to take place). 
4. Centrifuge at $500 \mathrm{rcf}$ for $15 \mathrm{~min}$ and transfer the supernatant in a new tube (see Note 16).

5. Centrifuge at $3500 \mathrm{rcf}$ for $15 \mathrm{~min}$ to retrieve microbes in the pellet (see Note 17).

6. Resuspend the pellet in $10 \mu \mathrm{L}$ PBS.

7. Inject third instar larvae (see Note 12) with $32.2 \mathrm{~nL}$ of the last solution obtained, using a Nanoject apparatus: the recipient larvae used are mutant for the putative opsonin gene.

8. Incubate for $60 \mathrm{~min}$ (see Note 13).

9. Bleed one larva per well, into $20 \mu \mathrm{L}$ of PBS on an 8-well microscope slide.

10. Incubate for $25 \mathrm{~min}$ in a humid chamber (see note $\mathbf{1 0}$ ).

11. Follow the steps described on sections 3.7 and 3.8.

\subsection{In/out differential immunostaining procedure for unlabeled microbes}

1. Fix hemocytes (from bled larva or adults) in 1\% paraformaldehyde in PBS for $10 \mathrm{~min}$, and fix dissected adult tissues in 4\% paraformaldehyde in PBS for 30 min, in a humid chamber (see Note 18).

2. Wash twice in PBS for 5 min.

3. Block for 30 min in a PBS solution with $2 \%$ Bovine Serum Albumin (BSA).

4. Stain the extracellular antigen with a primary antibody solution (against the microbe of interest), in PBS with $2 \% \mathrm{BSA}$ overnight at $4^{\circ} \mathrm{C}$. This antibody will not reach the intracellular antigen because the cells are not permeabilized at this step.

5. Wash twice in PBS for $5 \mathrm{~min}$.

6. Incubate with a FITC-labeled secondary antibody in a PBS solution with $2 \%$ BSA for $2 \mathrm{~h}$ at room temperature.

7. Wash twice in PBS for 5 min.

8. Permeabilize for $30 \mathrm{~min}$ in a PBS solution with $0.1 \%$ Triton $\mathrm{X}-100$ and $2 \%$ BSA (see Note 19). 
9. Stain the intracellular antigen with a solution containing the same primary antibody as used at step 4, in PBS with $0.1 \%$ Triton X-100 and 2\% BSA overnight at $4{ }^{\circ} \mathrm{C}$ (see Note 20 ).

10. Wash twice in PBS for 5 min.

11. Incubate with a Cy3-labeled secondary antibody in a PBS solution with $0.1 \%$ Triton X100 and $2 \%$ BSA for 2 hours at room temperature.

12. Wash twice in PBS for $5 \mathrm{~min}$.

13. Mount the slide in Vectashield with or without DAPI.

\subsection{Sample analysis by microscopy}

1. The samples are analyzed using a Zeiss Axioskope 2 fluorescent microscope or equivalent. In the case of red-fluorescent pHrodo-labeled particles, fluorescent microbes are considered as intracellularly located (see Note $\mathbf{2 1}$ ).

2. After in/out differential immunostaining, red and non-green particles are considered as intracellularly localized (see Note 22 and Figure 2). The number of red fluorescent bacteria that are not green fluorescent are counted in each DAPI-positive hemocyte.

3. Between 50 and 100 cells are analyzed per animal: the number of intracellular microbes is counted for each hemocyte (DAPI-positive or P1-positive, depending on the protocol), and the phagocytic index is calculated (\% of phagocytes containing at least 1 bacterium) $\mathrm{x}$ (mean number of bacteria per positive cell). After several independent experiments, each using at least 6 animals per condition, the non-parametric Mann-Whitney test is used for statistical analysis. 


\section{Notes}

\section{Antibodies against microbes}

The antibodies against microbes can work only if they can recognize surface epitopes: indeed, they should bind their epitopes even in the absence of permeabilization, a condition required for the in/out differential immunostaining procedure.

\section{Killed versus live microbes}

Killing procedure can affect microbial surface. UV and X-ray killing better preserve the surface properties of microbes than chemical fixation and heating $(24,25)$. Killed microbes may be more efficiently engulfed as compared to live ones, because some microbes have established active mechanisms to escape detection or engulfment by phagocytes: some of these mechanisms may be affected when microbes are killed. It is what we clearly found for $P$. aeruginosa $(5,16)$.

\section{The pHrodo labeling needs to use live plasmatocytes}

The pHrodo technique is performed using live plasmatocytes as fixation leads to the loss of the fluorescent signal. Thus, it is fast but requires immediate analysis of the samples. If one fails to observe any red signal in a mutant background, it does not imply that internalization is blocked, as only the maturation step might be affected. In such cases, it is therefore valuable to use the other techniques that allow monitoring internalization such as FITC-labeled microorganisms and quenching of the extracellular bacteria or the in/out differential staining procedure.

\section{Dose of microbes to use in phagocytosis assays}


Depending on the phagocytosis efficiency of a given microbe, it is necessary to determine the optimal dose to use. For instance, we observed that E. coli and S. aureus are much more efficiently phagocytosed as compared to $S$. marcescens and P. aeruginosa.

\section{Addition of Tween 20 in fungal solutions and sedimentation}

A low concentration of Tween 20 helps preventing fungal particle aggregation. A PBS solution without Tween 20 is used in the case of bacterial solutions. Before injection, fungal solutions need to be mixed because of the fast sedimentation of fungi. This is less critical for bacterial solutions that settle down much slower.

\section{Retrieved hemocytes from animal bleeding are mainly the circulating ones}

Among the two populations of larval hemocytes, the circulating ones are mainly retrieved by bleeding and the sessile ones are mainly lost, adherent to the larval tissues. Since adult hemocytes are mainly sessile, a similar population of cells is likely retrieved by bleeding or observed after adult tissue dissection.

\section{Larvae need to be synchronized}

It is critical to retrieve hemocytes from the third larval stage just after the peak of ecdysone expression that occurs at the mid-third instar and changes the behavior of larvae. The larvae have to be taken when they are out of the food (wandering stage) and before they stop to move. Even if the larvae are well synchronized, some can develop slower, especially if their density is too high. In fact, exposure to ecdysone is required for the hemocyte phagocytic activity and can thus modify the level of phagocytosis (26).

\section{Ex vivo versus in vivo phagocytosis assay}


This ex vivo procedure can be less accurate as compared to in vivo phagocytosis assay for two reasons:

- $\quad$ The composition of the hemolymph is different than PBS, and the buffer used may change the cell physiology, blocking some biological processes like engulfment. However, in our experience, ex vivo phagocytosis assays in PBS or Schneider's Drosophila medium yield similar results.

- $\quad$ Other organs (like the fat body) may secrete some factors that may influence the phagocytosis efficiency (for instance, opsonins), that may be required for the detection of some microbes by phagocytes.

\section{Larval versus adult hemocytes}

In adult flies, there are much fewer hemocytes retrieved (tens per fly) as compared to larvae (hundreds per larva), and the hemocytes are more autofluorescent. In addition, it is more time consuming to retrieve adult hemolymph than the larval one (see Figure 2F). However, in some cases, it may be more relevant to study phagocytosis by adult hemocytes, as one cannot formally exclude that the properties of hemocytes differ at both developmental stages. Indeed, most adult hemocytes are sessile: their attachment to tissues may modify the efficiency with which they are able to interact with microbes. Of note, we never tested adult hemocytes on ex vivo phagocytosis assays, only on in vivo assays, but it should be feasible.

\section{Incubation time to allow the hemocytes to settle down}

A humid chamber is important to avoid desiccation of the samples. During this incubation time, hemocytes will settle down. In the case of ex vivo assays, they will engulf microbes at the same time. In the case of in vivo assays on adult dissected tissues, this incubation time is not needed. 


\section{Trypan blue can quench the FITC fluorescence from extracellular particles}

Trypan blue is needed to quench the FITC fluorescence of extracellular particles. Thus, only the intracellular particles are able to emit fluorescence. This protocol allows quantifying the engulfment of particles.

\section{Larval injection}

Larval injection is not easy. To avoid killing the larvae, use a capillary as thin as possible. In addition, the larvae can be easily stuck on a dry surface (for instance on a glass microscopy slide) after making the larva itself dryer (by absorbing liquid from its surface on a paper). One may use double sided tape stuck on a slide. Then, the most convenient way to inject is to keep a position for the capillary almost parallel to the larval antero-posterior axis, and to inject in the middle of the body. Tweezers can be helpful to keep the larva immobilized (see Figure 2A).

\section{Incubation time for in vivo phagocytosis}

During this one-hour incubation step, the hemocytes in injected larvae or adult flies will engulf microbes.

\section{Monitoring phagocytosis in situ}

To monitor in vivo phagocytosis, one possibility is to bleed the injected insect using the same techniques as for the ex vivo assays. The other option is to dissect the tissues to visualize the hemocytes in situ, a strategy that requires the observation of multiple adult flies to obtain high enough numbers for meaningful statistical analysis. 


\section{Type of samples to use in opsonization assays}

The different samples to compare in an opsonization assay may be WT larvae to larvae mutant for a gene encoding a putative opsonin, for instance Tep4 (16). As control conditions, one can use flies deficient for phagocytosis, either by "latex" bead injection in WT flies or by using mutant flies impaired for phagocytosis $(4,14,15)$.

\section{Low-speed centrifugation}

Bacteria bound by opsonins are retrieved in the supernatant. The low-speed of centrifugation is essential to ensure that bacteria are not also pelleted with cells and tissues. This step was done successfully with bacteria but will need to be optimized for fungal spores or yeasts, which are bigger and sediment faster.

\section{High-speed centrifugation}

At this step, microbes bound to potential opsonins are retrieved in the pellet.

\section{Immunofluorescence troubleshooting with permeabilization}

One may observe some intracellular staining even when cells are not permeabilized. This is because formaldehyde fixation can affect membrane integrity (27). To avoid this, it might be better to use a $1 \%$ diluted formaldehyde solution and to shorten the period of fixation.

\section{Permeabilization of cell membranes}

Triton X-100 or NP-40 are harsh detergents, as compared as Tween 20, saponin or digitonin that are mild ones. Although an efficient permeabilization can significantly improve antibody access to antigens in the cytoplasm, a harsh detergent may disrupt some antigens. Triton X100 was suitable for all antigens described here. 


\section{Immunostaining with additional antibodies}

After the permeabilization step following the first antibody staining, if needed, it is possible to simultaneously use an additional primary antibody raised in another animal species, different from the anti-microbe antibody. Then, a secondary antibody labeled with another fluorescent dye can be used on the next step. If the antibody was raised in the same animal from the antimicrobe antibody, for instance in the case of the plasmatocyte-specific mouse P1 antibody, the whole procedure can be done in parallel, to get yellow plasmatocytes and yellow extracellular microbes (see Figure 2E). The fact that we cannot discriminate the color is not a problem to count engulfed particles.

\section{Fluorescence detection on pHrodo-labeled microbes}

pHrodo exhibits increasing fluorescence (red or green) as the $\mathrm{pH}$ becomes more acidic, such as that encountered in the mature phagolysosome. Thus, every fluorescent microbe can be considered as internalized by hemocytes into matured phagolysosome. Of note, as contrary as other protocols described here, the usage of pHrodo will not help to quantify every engulfed particle, but only the ones present in maturing phagolysosomes. In practical, there is always a weak background fluorescence, but its intensity is much lower than the signal from engulfed microbes.

\section{Fluorescence detection of microbes after in/out differential immunostaining}

The detected fluorescence of extracellular microbes after permeabilization is often only due to the first immunostaining step. This may be due to the saturation of epitopes during the first immunostaining step (before permeabilization), competing for a possible staining on the second immunostaining step (see Figure 2F). Of note, the secondary antibodies may be 
inverted between the first step and the second step of immunostaining (compare Figure 2C to Figure 2E and Figure2F). 


\section{References}

1. Lemaitre B, Hoffmann J. The Host Defense of Drosophila melanogaster. Annu Rev Immunol. 2007 Jan 2;25:697-743.

2. Gold KS, Brückner K. Macrophages and cellular immunity in Drosophila melanogaster. Semin Immunol. 2015 Dec 1;27(6):357-68.

3. Lanot R, Zachary D, Holder F, Meister M. Postembryonic hematopoiesis in Drosophila. Dev Biol. 2001 Feb 15;230:243-57.

4. Kocks C, Cho JH, Nehme N, Ulvila J, Pearson AM, Meister M, et al. Eater, a transmembrane protein mediating phagocytosis of bacterial pathogens in Drosophila. Cell. 2005 Oct 21;123:335-46.

5. Nehme NT, Liegeois S, Kele B, Giammarinaro P, Pradel E, Hoffmann JA, et al. A Model of Bacterial Intestinal Infections in Drosophila melanogaster. PLoS Pathog. 2007 Nov 23;3:e173.

6. Limmer S, Haller S, Drenkard E, Lee J, Yu S, Kocks C, et al. Pseudomonas aeruginosa RhlR is required to neutralize the cellular immune response in a Drosophila melanogaster oral infection model. Proc Natl Acad Sci U A. 2011 Oct 18;108:17378-83. 7. Nehme NT, Quintin J, Cho JH, Lee J, Lafarge MC, Kocks C, et al. Relative roles of the cellular and humoral responses in the Drosophila host defense against three gram-positive bacterial infections. PLoS One. 2011;6:e14743.

8. Quintin J, Asmar J, Matskevich AA, Lafarge MC, Ferrandon D. The Drosophila Toll Pathway Controls but Does Not Clear Candida glabrata Infections. J Immunol. 2013 Mar 15;190:2818-27.

9. Pearson AM, Baksa K, Rämet M, Protas M, McKee M, Brown D, et al. Identification of cytoskeletal regulatory proteins required for efficient phagocytosis in Drosophila. Microbes Infect. 2003 Aug;5(10):815-24.

10. Elrod-Erickson M, Mishra S, Schneider D. Interactions between the cellular and humoral immune responses in Drosophila. Curr Biol. 2000;10:781-4.

11. Nazario-Toole AE, Wu LP. Assessing the Cellular Immune Response of the Fruit Fly, Drosophila melanogaster, Using an In Vivo Phagocytosis Assay. J Vis Exp JoVE. 2019 Apr $10 ;(146)$.

12. Hoffmann D. [Role of phagocytosis and soluble antibacterial factors in experimental immunization of Locusta migratoria]. C R Acad Sci Hebd Seances Acad Sci D. 1976 Mar 8;282:1021-4.

13. Rutschmann S, Kilinc A, Ferrandon D. The Toll pathway is required for resistance to Gram-positive bacterial infections in Drosophila. J Immunol. 2002;168:1542-6.

14. Defaye A, Evans I, Crozatier M, Wood W, Lemaitre B, Leulier F. Genetic ablation of Drosophila phagocytes reveals their contribution to both development and resistance to bacterial infections. J Innate Immun. 2009;1:322-34.

15. Charroux B, Royet J. Elimination of plasmatocytes by targeted apoptosis reveals their role in multiple aspects of the Drosophila immune response. Proc Natl Acad Sci U A. 2009 Jun 16;106:9797-802.

16. Haller S, Franchet A, Hakkim A, Chen J, Drenkard E, Yu S, et al. Quorum-sensing regulator RhlR but not its autoinducer RhlI enables Pseudomonas to evade opsonization. EMBO Rep. 2018;19(5).

17. Avet-Rochex A, Bergeret E, Attree I, Meister M, Fauvarque MO. Suppression of Drosophila cellular immunity by directed expression of the ExoS toxin GAP domain of Pseudomonas aeruginosa. Cell Microbiol. 2005 Jun;7:799-810. 
18. Avet-Rochex A, Perrin J, Bergeret E, Fauvarque MO. Rac2 is a major actor of Drosophila resistance to Pseudomonas aeruginosa acting in phagocytic cells. Genes Cells. 2007 Oct; 12:1193-204.

19. Sinenko SA, Mathey-Prevot B. Increased expression of Drosophila tetraspanin, Tsp68C, suppresses the abnormal proliferation of ytr-deficient and Ras/Raf-activated hemocytes. Oncogene. 2004 Dec 2;23:9120-8.

20. Haller S, Limmer S, Ferrandon D. Assessing Pseudomonas virulence with a nonmammalian host: Drosophila melanogaster. Methods Mol Biol. 2014;1149:723-40.

21. Lestradet M, Lee K-Z, Ferrandon D. Drosophila as a model for intestinal infections. Methods Mol Biol Clifton NJ. 2014;1197:11-40.

22. Kurucz E, Markus R, Zsamboki J, Folkl-Medzihradszky K, Darula Z, Vilmos P, et al. Nimrod, a putative phagocytosis receptor with EGF repeats in Drosophila plasmatocytes. Curr Biol. 2007 Apr 3;17:649-54.

23. Bosch PS, Makhijani K, Herboso L, Gold KS, Baginsky R, Woodcock KJ, et al. Blood cells of adult Drosophila do not expand, but control survival after bacterial infection by induction of Drosocin around their reservoir at the respiratory epithelia. bioRxiv. 2019 Mar $16 ; 578864$.

24. Datta SK, Okamoto S, Hayashi T, Shin SS, Mihajlov I, Fermin A, et al. Vaccination with irradiated Listeria induces protective T cell immunity. Immunity. 2006 Jul;25:143-52.

25. Wheeler RT, Fink GR. A Drug-Sensitive Genetic Network Masks Fungi from the Immune System. PLOS Pathog. 2006 Apr 28;2(4):e35.

26. Regan JC, Brandão AS, Leitão AB, Mantas Dias AR, Sucena E, Jacinto A, et al. Steroid hormone signaling is essential to regulate innate immune cells and fight bacterial infection in Drosophila. PLoS Pathog. 2013 Oct;9(10):e1003720.

27. Pelts M, Pandya SM, Oh CJ, Model MA. Thickness profiling of formaldehyde-fixed cells by transmission-through-dye microscopy. BioTechniques. 2011 Jun 1;50(6):389-96.

\section{Figure legends}

\section{Figure 1: Overview of the different phagocytosis and opsonization assays}

A, phagocytosis assays. This scheme shows the main steps that allow quantifying phagocytosis ex vivo (left) and in vivo (right). Numbers in red indicate the corresponding section in the text.

B, opsonization assays. This scheme shows the main steps of the opsonization assay.

Indications in red allow finding the corresponding section in the text to read the full description of the procedure. 


\section{Figure 2: Illustrations of in vivo phagocytosis assays in D. melanogaster larvae and}

adults

A, B, C: in vivo phagocytosis assays in larvae. A, Image illustrating the procedure of larval infection by injection, showing the position of the capillary (right) and the help of tweezers; the dried larva is stuck to double sided tape on a slide. B, Microscopy image of killed bacteria (labeled with red pHrodo) in a GFP-expressing plasmatocyte (from hmldelta-Gal4,UAS-GFP flies). C, Microscopy image of bacteria with hemocytes, after in/out differential immunostaining: bacteria were first stained in green before permeabilization and then in red. Bacteria engulfed by hemocytes can only be stained in red, whereas extracellular bacteria are both red and green (yellow); the nucleus of an hemocyte is stained in blue (DAPI); the plasmatocyte membrane is outlined.

D, E, F: in vivo phagocytosis assays in adults. D, Image illustrating the procedure of adult fly infection by injection. E: Microscopy image of yeasts with hemocytes, after in/out differential immunostaining. Adult Drosophila tissues were dissected and fixed after $C$. glabrata injection. Hemocytes are shown in purple (P1 antibody). Yeasts cells were first stained in red before permeabilization and then in green. Ingested yeasts can only be stained in green, whereas extracellular yeasts are both green and red (yellow). F, left: adaptor for long capillaries with a syringe after a flexible tube, for adult hemolymph collection; middle: image illustrating the procedure of adult hemolymph collection by flooding the thorax with a large volume of PBS (about $20 \mu \mathrm{L}$ ), using the adaptor shown at the left image - hemocytes are collected in PBS on a 8-well microscope slide; right: Metarhizium anisopliae injection experiment: microscopy image of fungi with hemocytes, following in/out differential immunostaining procedure: fungi were first stained in red before permeabilization and then in green. Ingested spores can only be stained in green, whereas extracellular spores are both green and red (yellow); the nucleus of an hemocyte is stained in blue (DAPI). 


\section{Acknowledgments}

We would like to thank François Lapraz (Institute of Biology Valrose, Nice, France) and Gábor Csordás (Institute for Genetics, University of Cologne) for their advice for adult hemolymph collection, and Marion Draheim for critical reading of the manuscript. DF's team work at SFHI is partially funded through the 1,000 Talent Program of Global experts of China. 


\section{A - Phagocytosis assays}

ex vivo

(3.4)

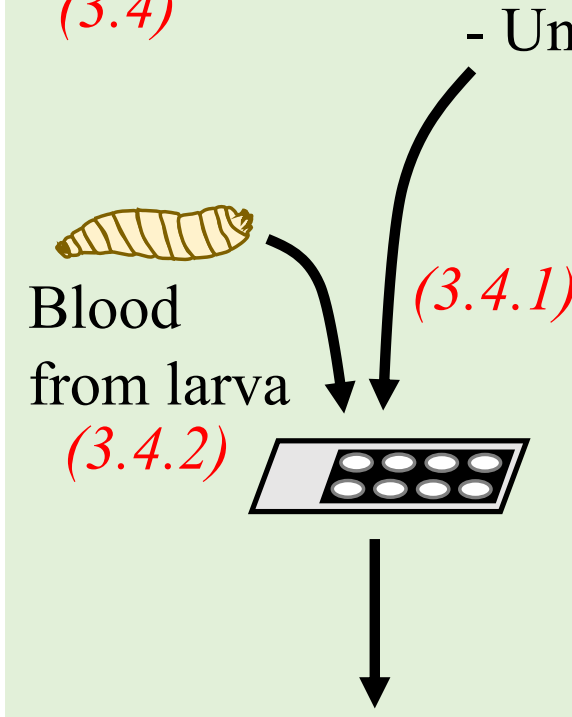

Incubation

Cell settle down

(3.4.3)

- pHrodo-labeled killed microbe

- Unlabeled live microbe

(3.2)
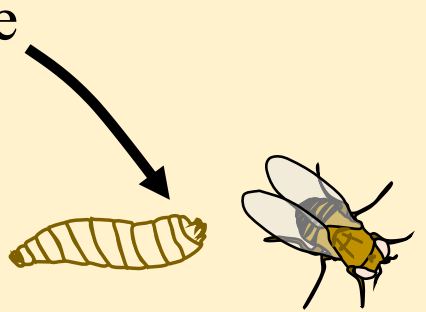

Injection in larvae or adults (3.5.1)

Incubation

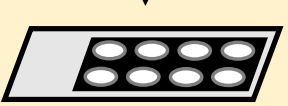

(3.5.2)

Bleed or adult tissue dissection

\section{(3.5.3) $\downarrow$}

Cell settle down

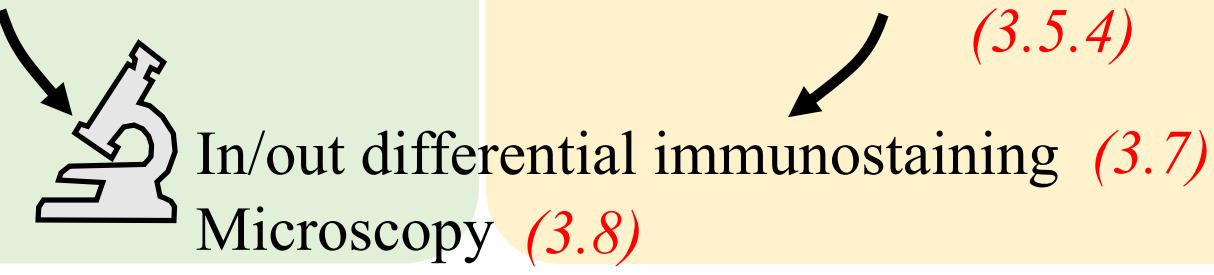

\section{B - Opsonization assay (3.6)}

Hemolymph from

WT larvae

(3.6.2)

(3.6.3 to 3.6.6)

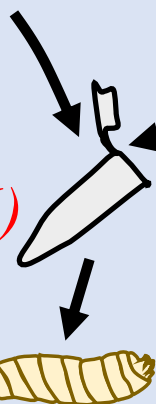

8888Bleed

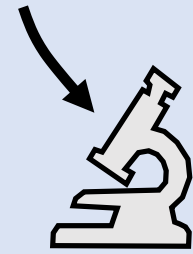

Injecti

Live
microbe

Hemolymph from putative

(3.6.1) opsonin mutant larvae

$$
\downarrow \text { Incubation (3.6.8) Incubation } \downarrow
$$

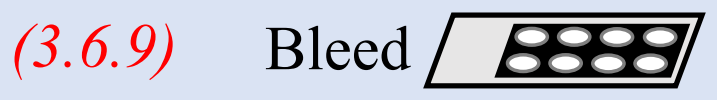

Cell settle down (3.6.10)

$(3.6 .3$ to 3.6 .6$)$

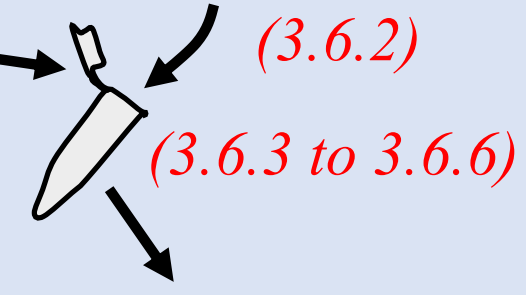



A Larval assay
B

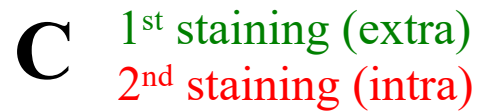
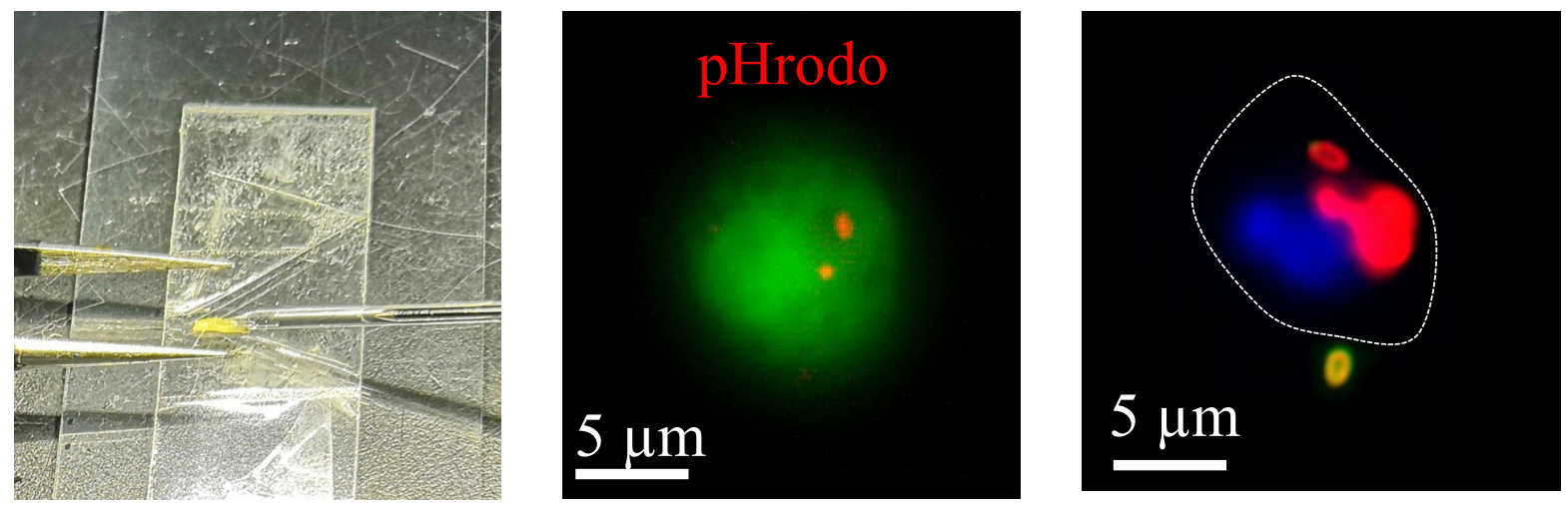

\section{Adult assay}

E Live Candida in adult tissue
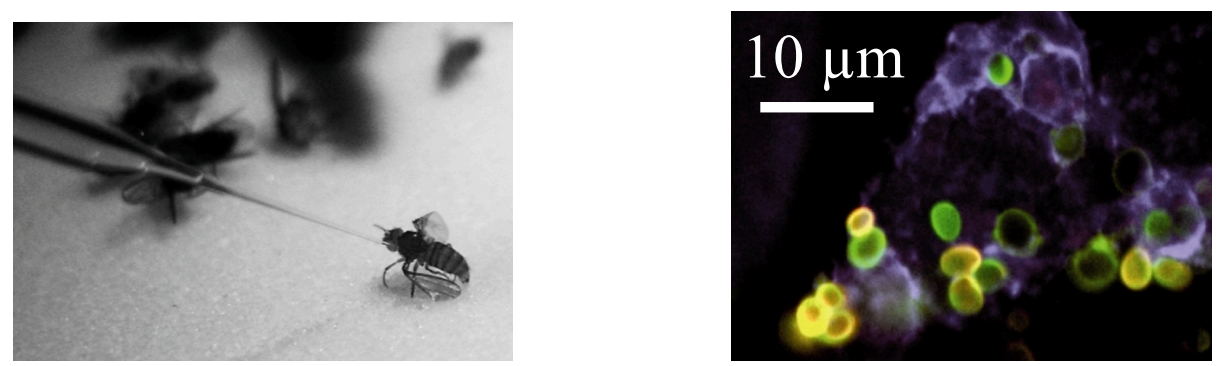

$1^{\text {st }}$ staining (extra) $2^{\text {nd }}$ staining (intra)

Hemocyte

F Live Metarhizium in adult flies

$1^{\text {st }}$ staining (extra) $2^{\text {nd }}$ staining (intra)
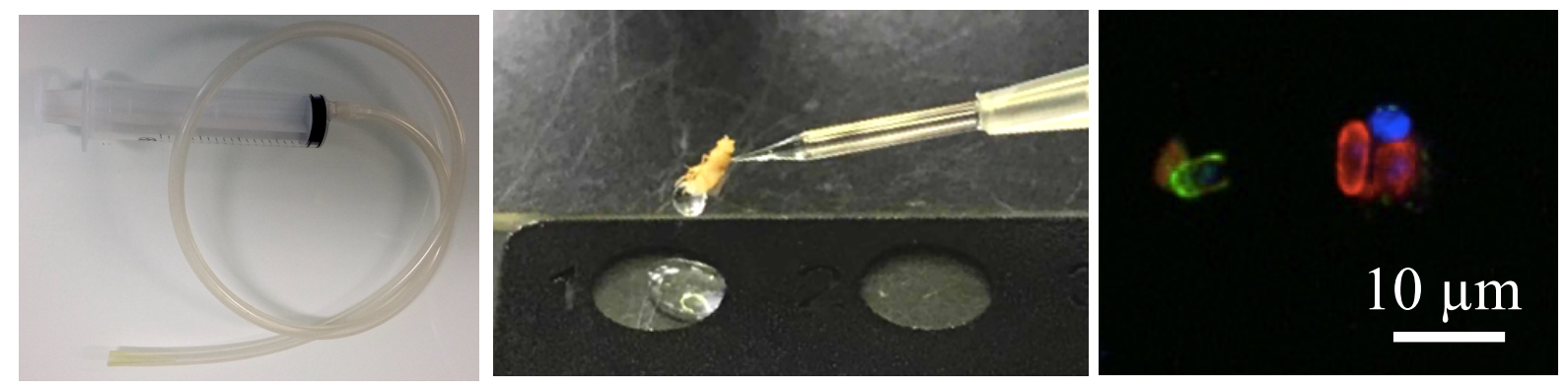

\section{Figure 2}

